\title{
Modeling and verification of the Multi-connection Tactile Internet Protocol
}

\author{
Delia Rico \\ ITIS Software, Universidad de Málaga \\ Málaga, Spain \\ deliarico@uma.es
}

\author{
María-del-Mar Gallardo \\ ITIS Software, Universidad de Málaga \\ Málaga, Spain \\ mdgallardo@uma.es
}

\author{
Pedro Merino \\ ITIS Software, Universidad de Málaga \\ Málaga, Spain \\ pmerino@uma.es
}

\begin{abstract}
Tactile Internet (TI) refers to the transmission of touch and the real-time control of applications like the remote control for teleoperation of machines, drones or vehicles. Traditional TCP and UDP protocols are not suitable for these applications, because they are mainly designed for content delivery or non real-time interactive applications. Some ideas from transport protocols for real-time multimedia like RTP are closer to Tactile Internet; however, they do not satisfy requirements of low latency and high reliability. In this paper, we present the Multi-connection Tactile Internet Protocol (MTIP), a novel transport protocol on top of the Internet Protocol to support the reliability and latency for Internet applications over multi-homed devices connected to several wireless networks. In order to provide that service, MTIP is based on the combination of sequence numbers and timestamps in packets, enhanced with global clocks and context awareness. The paper focuses on building a state machine based model of MTIP to prove the correct behaviour of the protocol using the sPIN tool to verify a number of relevant correctness properties.
\end{abstract}

\section{CCS CONCEPTS}

- Networks $\rightarrow$ Transport protocols; • Theory of computation $\rightarrow$ Formal languages and automata theory.

\section{KEYWORDS}

Formal Modeling, Transport Protocol, Multiconnectivity, Tactile Internet, Automatic Verification

\section{ACM Reference Format:}

Delia Rico, María-del-Mar Gallardo, and Pedro Merino. 2021. Modeling and verification of the Multi-connection Tactile Internet Protocol. In Proceedings of the 17th ACM Symposium on QoS and Security for Wireless and Mobile Networks (Q2SWinet '21), November 22-26, 2021, Alicante, Spain. ACM, New York, NY, USA, 10 pages. https://doi.org/10.1145/3479242.3487328

\section{INTRODUCTION}

In the paper introducing the IEEE "Tactile Internet" (TI) Standards working group (WG) [13], Tactile Internet is defined as "A network for remotely accessing, perceiving, manipulating, or controlling real or virtual objects or processes in perceived real time by humans or

Permission to make digital or hard copies of part or all of this work for personal or classroom use is granted without fee provided that copies are not made or distributed for profit or commercial advantage and that copies bear this notice and the full citation on the first page. Copyrights for third-party components of this work must be honored

For all other uses, contact the owner/author(s).

Q2SWinet '21, November 22-26, 2021, Aliante, Spain.

(c) 2021 Copyright held by the owner/author(s).

ACM ISBN 978-1-4503-9080-4/21/11.

https://doi.org/10.1145/3479242.3487328 machines". Such definition includes use cases like teleoperation, automotive, immersive virtual reality, operation of drones, advanced interpersonal communications (holograms), live haptic-enabled broadcast or cooperative automated driving. These use cases require, in most cases, wireless communications due to the mobile nature of the devices involved at the end points.

In terms of traffic patterns, as detailed in [13], TI requires latencies end to end below $5 \mathrm{msec}$ (e.g. Immersive virtual reality) or even close to $1 \mathrm{msec}$ (e.g. Haptic feedback). Required reliability is usually 99.999\%; however, this value will be higher for use cases such a live haptic-enabled applications. Traffic is usually sent as bursts of a few bytes or hundreds of bytes, and the average data rate ranges from 1 to $100 \mathrm{Mbps}$.

It is clear that traditional TCP and UDP protocols cannot support the TI requirements. For instance, TCP can only offer $100 \%$ reliability, often causing a long latency due to the bad quality of the path between the end-points. TI applications can work with some packet loss at the application layer, provided that a set of packets arrives on time. We call this feature partial reliability. UDP does not add any specific quality to the underlying path, so we cannot expect any reliability improvement. Other protocols that provide contributions that come closer to the TI requirements are the Real-time Transport Protocol (RTP) [24] and extensions like the Multipath RTP (MPRTP) [26]. However, these protocols are focused on providing service for real-time multimedia with typically high amounts of data, instead of controlling limited packets with very strict constrained deadlines like in TI remote operations and sensing.

We foresee a TI scenario where most of the devices will be connected to several $5 \mathrm{G}$ and other wireless networks at the same time in a private or public area. In this scenario, we present the Multiconnection Tactile Internet Protocol (MTIP), a novel transport protocol to offer programmable quality to applications that need a dynamic compromise between latency, reliability, throughput and jitter, among other parameters. MTIP combines the management of multiple interfaces and paths with context awareness to provide a master-slave communication of sequenced deadline-aware packets with high (but partial) reliability and low latency. The use of multiple interfaces allows sending several copies of the message using the selection of the best subset of paths at the time of sending.

The main challenge at this stage is to guarantee the correctness of the algorithms to send and to receive packets. Following the huge amount of work done in formal modeling and verification of communication protocols, in the paper we adopt the sPIN framework for formal modelling and verification of the new protocol. Once the protocol has been proven to be correct from the algorithm's perspective, we will address performance issues in further work. 
The rest of the paper is organized as follows. Section 2 presents related work. Section 3 provides an overview of MTIP's requirements, general architecture and data management procedures. Section 4 presents the work on the formal modelling and verification of the protocol. Finally, Section 5 contains the conclusions and future work.

\section{RELATED WORK}

Some transport protocols reuse the existing IP networks with the same objective as MTIP in the areas related to Tactile Internet. The Interactive Real-Time Protocol (IRTP) [7] is a connection oriented protocol for remote control of robots inspired by TCP, but that also uses an unreliable mode to avoid packet loss being retransmitted. The Efficient Transport Protocol (ETP) [30] separates the two flows in the end to end communication to reduce the round trip time by adapting the inter packet gap to the network congestion conditions. Gokhale et al. [15] presents an application layer protocol with a similar aim: to enhance haptic communications over UDP and IP. It uses adaptive sampling and transmission rate control, among others, to improve the communication in terms of latency. Further relevant protocols can be found in the survey by Kokkonis et al. [20].

Compared to these protocols, MTIP provides more mechanisms for reliability using multiple copies in several paths to increase the chances for the packet to arrive within the deadline. In addition, it uses application preferences and reports the network status to the application to exploit awareness.

Multipath TCP (MPTCP) [14], QUIC [18] and its multipath version, Multipath QUIC (MPQUIC) [27] are protocols that do not focus on TI but are relevant in current Internet evolution. They are mature solutions to exploit multiple paths with a single network interface or in a multi-homed devices. They are designed to transmit big amounts of available data, where control flow and congestion control are mandatory. MTIP's scope is not the same as that of these protocols. MTIP is a real time protocol for remote control and sensing, where traditional congestion control methods are not needed and can even be counterproductive. MTIP is more comparable to solutions like Multipath RTP (MPRTP) [26]. However, there are some differences between them, the most important being that the timestamps in RTP are used for reordering, not for deadline control, and the fact that MPRTP is focused on real-time multimedia while MTIP is focused on remote operation and sensing.

There are also long term proposals to replace the current Internet architecture with new ones for the TI, like FlexNGIA [33], which are not comparable to MTIP. In [23], we present a survey of more end to end solutions to improve latency and reliability over $5 \mathrm{G}$ networks, many of them proposing major changes in the network.

Regarding formal modeling and verification of related protocols, there are some works using models for analysing multipath protocols. For instance, Zhang et al. [32] work on a QoE-driven
MPTCP-based data delivery model and use a Markov model to predict mobile node connections. However, the model they present is not aimed at carrying a formal verification of the protocol operation itself. To our knowledge, only a few research works have addressed the formal verification of protocols focused on TI aspects. Chouali et al. [8] present the formal verification of a new data protocol for Connected Vehicles. The protocol is a variation of the publish subscribe operation of the MQTT protocol and the contribution focuses its efforts on data workload reduction at the processing infrastructure. Dombrowski et al. [11] perform model-checking verification of a MAC layer protocol for Ultra-Reliable Low-Latency Wireless Networks. They study the protocol's performance and correctness, defending the use of Probabilistic Timed Automatas for the analysis of time-dependent protocols that are subject to random effects. Finally, Cai et al. [6] conduct the formal verification of a control protocol for Cyber-Physical Systems. Their aim is to model and verify the correctness operation of the control of remote devices in Wireless Sensor Networks.

We contribute to this work on formal modelling and verification with the use of the PROMELA language and the SPIN [17] tool to check the correctness of this novel protocol. Promela (Protocol Meta Language) was specifically designed for modeling network protocols and algorithms, and the tool sPIN has been used in a number of academic and industrial applications. In Section 4, we provide more details on this tool.

\section{MTIP PROTOCOL}

\subsection{Tactile Internet requirements}

TI use cases rely on the basic idea of aiming for perceived real time with strict deadlines and optional support of reliability [13]; however, each type of application has its concrete considerations as presented in [16] or [19]. In fact, additional international standardization organizations, such as the $3 \mathrm{GPP}$, are also defining concrete requirements of TI-like applications through 3GPP Cyber-Physical Systems [4] and service requirements for the $5 \mathrm{G}$ systems [1,2].

To give some examples, in in Table 1 we compare two TI use cases: Industrial Control, also referred to as teleoperation or discrete automation; and the Internet of Drones, also referred to as Unmanned Aerial Vehicle control or, in some cases, as part of Intelligent Transportation Systems. As can be seen, data rates are limited, but with application specific requirements on latency and reliability, depending on the environment. There are also concrete requirements as limited jitter in Industrial Control, or wireless support in Drone Operation. These limited data rates open up the possibility of using redundancy in several paths to meet the specific requirements of the use cases with a possible increase of reliability, if needed. If reliability is increased in that way, then flow control and retransmissions can be avoided to reduce latency. These are key ideas behind MTIP.

Table 1: Comparison of Remote Operation Tactile Internet Use Cases

\begin{tabular}{|c|c|c|c|c|c|c|c|c|c|}
\hline Use Case & Latency & Reliability & Environment & Service Area & Packet Size & Data Rate & Communication Type & Other Requirements & Refs \\
\hline Industrial Control & $\begin{array}{c}1-10 \mathrm{~ms} \\
10-100 \mathrm{~ms}\end{array}$ & $\begin{array}{c}99.99 \%-99.9999 \% \\
99.999 \%\end{array}$ & $\begin{array}{c}\text { Highly dynamic } \\
\text { Dynamic }\end{array}$ & Lower $(100 / 0 \times 100 / 0 \times 30 \mathrm{~m})$ & Small (40 - 250bytes) & Small (1 - 10Mbps) & Master $<->$ Slave & 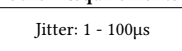 & $\begin{array}{l}\text { IEEE }[16], \\
3 \mathrm{GPP}[1,4]\end{array}$ \\
\hline \multirow{2}{*}{ Internet of Drones } & $1-5 \mathrm{~ms}$ & $99.9-99.999 \%$ & Kinesthetic (Closed Loop) & \multirow{2}{*}{ Higher (Several Kms) } & \multirow{2}{*}{ Small (40 - 250bytes) } & \multirow{2}{*}{ Small (1 - 10Mbps) } & \multirow{2}{*}{ Master $<->$ Slave } & \multirow{2}{*}{ Just Wireless } & IEEE [16], \\
\hline & $10-100 \mathrm{~ms}$ & $99.9-99.999 \%$ & Tactile (Open Loop) & & & & & & $3 \mathrm{GPP}[1,2]$ \\
\hline
\end{tabular}




\subsection{MTIP basis}

MTIP is designed to support communication between multi-homed master and slave TI nodes over several wireless communication networks. TI applications send short messages, usually following a periodic pattern defined by the control loops at the master and the slave. The flows of orders (master to slave) or status information (slave to master) are usually independent flows per direction, even if they share the same MTIP layer.

The key concept in MTIP is the link (multi-connection) between the two protocol entities built on top of several IP interfaces. Two sets of network interfaces (one set on each side) defines the access network technologies and the path between the end points, which can vary over time in terms of the Key Performance Indicators (KPIs). Figure 1 represents how MTIP is located in the TCP/IP protocol family to support such end-to-end links.

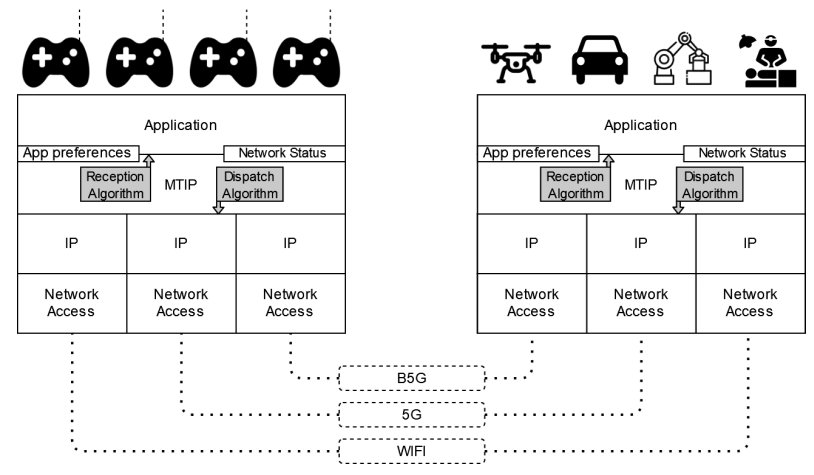

Figure 1: Sample scenario for MTIP applications

In practice, one link is a set of sublinks with multi-homed devices as end points. As Qadir et al. [22] noted, the Internet's future is inherently multipath. Multihoming capable devices are growing, as well as interface diversity. This creates a clear path towards multi-connectivity. The use of multiple paths to take advantage of each path's characteristics and to achieve enhanced reliability or throughput aggregating resources can help increase the quality of experience levels to reach current requirements. The benefits of multiple connectivity include better reliability, network offloading, improved availability, etc.

In MTIP, the default "Reception" and "Dispatch" algorithms exploit the information from both the application and the sublinks. As shown in Figure 1, the master and the slave applications periodically expose the preferences on how to process the traffic following the Intent Based Networking paradigm [9]. In parallel, both algorithms use information of the current state of the sublinks thanks to online measurement information. The status of the sublinks can also be exposed to the application.

MTIP relies on sequence numbers and timestamps in the packets to control its operation. Sequence numbers enable the scheduling of network interfaces in the source, including redundancy using several interfaces for the same packet, and the reassembling of packets in the destination, managing losses and reordering. We assume that some global synchronization is in place, so a timestamp can be directly used in the packets to control the deadline before sending the payload to the applications. Such global clocks can be provided by the wireless network or with external processes like the Global Navigation Satellite System (GNSS), the Precision Time Protocol (PTP) [25], the Network Time Protocol (NTP) [21], etc., as done with the RTP clock control [29]. The use of both sequence numbers and timestamps allows detecting duplicated and obsolete packets, providing to the application the packets that are on time without reorder.

It is worth noting that, like other protocols for real-time communication, MTIP does not define explicit methods for flow control or congestion control beyond ensuring the order of messages to the applications. There are two main reasons for that decision. On one hand, including artificial waiting periods for the packets could negatively impact the low latency needs for TI. On the other hand, the traffic patterns produced by TI applications are not like the content distribution applications, where application data are always available and can be sent with the sole limitation of the available bandwidth. To avoid issues when sending data without any specific stop and go mechanisms, the sequence numbers and timestamps are defined with 64 bits length to be reused unequivocally in a circular manner.

Regarding security, MTIP will trust the specific mechanism implemented in the wireless networks supporting the connections, such as ciphering in the air interface and secure GTP tunnels [3] in the fixed part of the network.

\subsection{Data transmission algorithms}

Data management in MTIP can be summarized with two features: a dispatch algorithm and a reception algorithm. In Figure 2, we present an example of a Message Sequence Chart (MSC) of a typical MTIP exchange (with the example of a maximum latency of $5 \mathrm{~ms}$ tolerated).

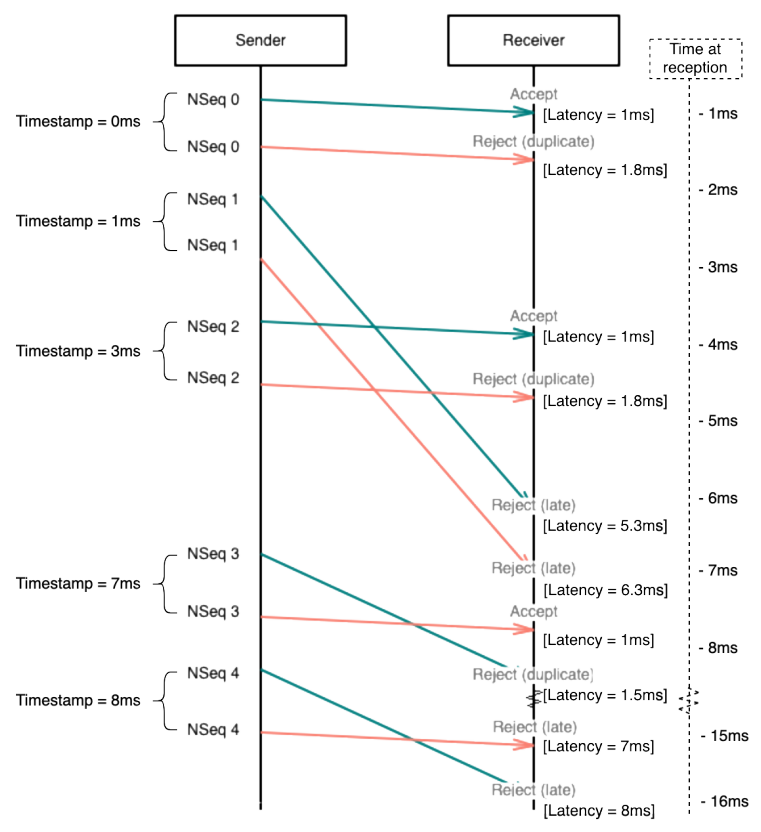

Figure 2: Example MSC of MTIP 


\subsubsection{Sending packets: dispatch algorithm.}

The dispatch algorithm's main function is to perform a continuous evaluation and selection of the concrete sublinks which are going to be used to send the redundant copies of messages. Ideally, every sublink would be used to send data, however, application can restrict it in the presented way so as to reduce overload. The algorithm uses application quality of service preferences and the network state measurements to select a concrete number of sublinks to send packets (more information about awareness in Section 3.4). Sublinks are ranked individually according to measured KPI values, after which MTIP selects the best sublinks in this ranking in terms of the KPIs selected by the application each time a new packet is sent. The outcome of the dispatch algorithm is always the selection of a fixed number of sublinks to send the redundant copies of a packet. Each copy of the same packet carries the same sequence number to control reordering, losses and duplicates; and the same timestamp, marking the exact time when the unique data was generated, to be able to calculate the deadline of the packets arriving on the reception side. The deadline or lifetime of a packet is calculated adding the timestamp of the moment the order is generated to the maximum latency tolerated, defined by application.

\subsubsection{Receiving packets: reception algorithm.}

When a data packet arrives, MTIP checks if the packet is relevant to the application before sending it to the upper layer. A packet is still relevant if it is not a previously received duplicate and if it has not met its deadline yet. In order to check this, the reception algorithm has the following mechanisms.

First of all, there is a window that controls the relevance of packets in terms of time. This window takes into consideration the maximum latency tolerated, defined by application preferences, to determine if the packet has met its deadline and should be discarded. Then, comparing the sequence numbers of the packets that are being received, the algorithm checks that just one packet of a series of duplicates is sent to the upper layer. The final step is intended to correct out of order. When out of order is detected through gaps in the sequence numbers, MTIP stores the packet for a certain amount of time before sending it to the application. The received packet waits until the missing packet arrives or until it is considered lost, using the information of the remaining life of the packet that is waiting (refer to [5] for further information about static/adaptive packet expiration). In any of the cases, the packet waiting is sent to the application layer before meeting its deadline.

\subsection{Awareness}

MTIP uses information from outside the scope of the transport layer to offer an enhanced service. This information is divided in two sections: network awareness and application awareness.

First, MTIP performs continuous measurements on the network to have updated information about the network state of each possible path (sublink). These measurements can be performed on-the-go, using the timestamps and sequence numbers on packets. However, the application has additional measurement mechanisms to force better characterization on demand of specific KPIs. The result of these different operations is a database with the characterization of each sublink in terms of the different KPIs. This information is used by the dispatch algorithm to select the sublinks that should be in use to send the redundant data.

MTIP is also aware of application preferences. The application can define different preferences to adapt the operation of the algorithms to its needs. It can decide the importance weight of the different KPIs, which are going to be used in the selection of sublinks to send data, and even concrete values as the maximum latency that should be tolerated on the reception side. The main KPIs in TI communication are latency and reliability; however, additional KPIs can be selected if the application considers them relevant for its operation. Moreover, the application can select protocol specific preferences to flexibly adjust the operation of the dispatch and reception algorithms, such as the number of redundant sublinks used to send data or if MTIP should try to wait for out of order packets or just skip that operation.

\section{FORMAL MODELING AND VERIFICATION OF MTIP}

When defining new transport protocols, the main complexity and the most potential errors are the mechanisms for accepting data in the proper order, controlling deadlines and controlling the flow to the application. Such problems were the main motivation of formal verification in past decades [28] and fortunately powerful tools are now available. In this section, we use formal models in PROMELA and temporal logic formulas to verify the correctness of MTIP with the model checker SPIN. The objective of the PROMELA models is to represent the abstraction of the dispatch and reception algorithms with the detail that allows to check a number of requirements related to the service offered to the application. The requirements of MTIP's logic can be summarized as "the application receives in order all data that is on time and that is not a duplicate". In order to use SPIN, this general property has been divided into 4 different smaller and easily verifiable properties:

- P1: Application never receives duplicates.

- P2: Application never receives packets that have met their deadline (packets that are late).

- P3: Application never receives out of order packets.

- P4: Every packet with a different sequence number (that is not late) is sent to application.

Before providing the details of the models we introduce SPIN and PROMELA.

\subsection{SPIN Model Checker and Promela Language}

SPIN is an open-source model checking tool developed at Bell Labs by Gerard Holzmann [17]. Model checking is an automatic verification approach able to automatically verify models of concurrent systems against their most relevant properties, usually specified in some variant of temporal logic [31]. A model checker is an automatic tool that typically accepts as inputs both a system model and a desirable property and returns yes if all behaviours of the model satisfy the property, or no if an incorrect behaviour has been found during the analysis. In this case, the tool also returns a counterexample, which is very useful to debug the model/system. 
The input language for models of SPIN is called PROMELA. The PROMELA syntax and semantics are based on those of $\mathrm{C}$, although some features that are not needed for the modeling tasks, such as floating point types or pointers, have been removed. In contrast, PROMELA includes other sentences (such as atomic instructions and non-determinism) which make it easier to construct system models. A typical PROMELA program is composed of a sequence of global types and variables such as the ones declared in Listing 1 and a set of processes that execute by interleaving their instructions. Processes can communicate using global shared variables or by means of channels which can be defined as synchronous or asynchronous. Processes are declared using the keyword proctype with a syntax is similar to $C$ functions, i. e., they can contain local types, variables and executable code. The difference is that when an instance process is created, it executes its code in new execution thread. Process instances may be directly declared and instanciated placing the active keyword before proctypes (line 14 in Listing 1). Alternatively, they can be instantiated using the run sentence in the init process which acts as the $\mathrm{C}$ main process.

The notion of executability is essential in PROMELA. An instruction is executable if it can be selected by the sPIN engine to be executed next. Executability is extensively used in the language to synchronize processes. If the program counter of a process is pointing to a non-executable instruction, the process is blocked until the sentence is executable. For instance, the Boolean expressions can be use as normal sentences, but they are executable only when they are true; as in line 39 of Listing 1, where (ExpectedNum!=SeqNum) blocks the sender() process until the expression is true.

Control sentences if/fi and do/od introduce non-determinism in the language. For example, the instruction if $/ \mathrm{fi}$ in Listing 6 (lines 5-9) is a typical non-deterministic selection in PROMELA. It has three branches (determined by symbols “::”), each one starting at an assignment to variable time. When an if/do instruction is to be executed, sPIN selects, in a non-deterministic manner, one branch among all the executable ones to continue the execution. A branch is executable if its first statement (the guard) is. In the example, as assignments are always executable, any branch can be selected, which means that after executing sentence if, variable time can have value 0,1 or 2 . If no branch of an if/do sentence is executable, then the process is suspended waiting for one of them to become executable in the future. It is also possible that an if/do sentence has an optional else branch, as the if sentence of Listing 6 in lines 12-20. In this case, the if sentence never suspends, since if no branch is executable, the program counter automatically jumps to the else branch.

It is also common to use receive or send operations on channels as branch guards in if/do sentences as shown in the if sentence of Listing 7 in lines 12-15. Naturally, a receive instruction on a channel is not executable if the channel is empty and, inversely, a send operation is not executable if the channel is full. In the example, process receiver is blocked at line 12 until a message can be read from channel sublink[0] or sublink[1]. As explained above, if both channels contain data, the branch to be executed is selected in a non-deterministic manner. Observe that PROMELA uses the CSP notation to read/write from/on channels.

Constructor atomic is used in Promela to define a sequence of process instructions as atomic which, in practice, means that
SPIN will not carry out a context switch during the execution of an atomic instruction unless the process blocks. For instance, in Listing 5, the atomic instruction between lines 11-16 means that after receiving data from channel source (in line 11), sPIN will try to successively update array timebuffer, send data through channels sublink[0] and sublink[1] and, finally, increase time. Observe that instruction (timebuffer [SeqNum]==FREE) acts as a guard in the atomic sentence, i.e., atomic blocks if timebuffer[SeqNum] is not FREE. Sentence d_step is similar to atomic except that it can only nest instructions which can never block. Another interesting PROMELA instruction is timeout. This sentence is handled by SPIN as a Boolean which true only when no other process instruction can be selected to continue the execution. Thus, timeout is used to avoid system deadlocks or to implement more complex behaviour as in Listing 3 in lines 21 and 25. In this code, timeout is used to first detect whether the expected data is ONTIME, whether it is LATE, or otherwise, whether the data has been lost, and the receiver should wait for another one.

sPIN tool is able to directly analyze several types of model properties such as deadlocks or assertions. The mechanism to analyze deadlock is somehow embedded in the tool, since it is assumed that no model should block. In addition, user can introduce assertions in the model code using assert. Listing 4 contains several assertions in lines 12 and 15. When the tool is traversing the state space produced by a model, if a false assertion is found, it is reported as an error.

\subsubsection{Specification of properties with Temporal Logic.}

As mentioned above, once a PROMELA model has been constructed, SPIN is able to automatically analyze their desirable properties written in the linear temporal logic (LTL). This logic is an extension of propositional logic with modal operators which enable the description of properties that occur along time in execution traces produced by the model under analysis. Each execution trace is composed of the sequence of observable system states (such as $\left.\pi=s_{0} \cdot s_{1} \cdot s_{2} \cdots\right)$ which represents a particular system execution. State $s_{0}$ is the initial system state, and $s_{i}(i>0)$ is constructed by applying a basic instruction (an assignment, the evaluation of a Boolean expression, a channel reading, and so on) to its previous state $s_{i-1}$. LTL assumes that all traces under analysis are infinite, so finite traces are transformed into infinite by repeating their last state indefinitely.

The syntax of LTL supported by SPIN is $\Phi=p|! \Phi| \Phi|| \Phi|[] \Phi|<>$ $\Phi \mid \Phi U \Phi$, where $p$ is an atomic proposition chosen from a set of predefined propositions. In the case of SPIN, atomic propositions are usually Boolean expressions constructed with model variables. They can also be statements of the form $P[\mathrm{~m}] @$ label which are true when the instance $m$ of process type $P$ is located at program counter labelled by label. The simpler version P@label may be used when there is only one instance of proctype $P$ in execution. Operators ! and $\|$ are the $C$ Boolean operators for the negation and disjunction of expressions. As usual, conjunction $\& \&$ or implication $->$ can be derived from from ! and $\|$.

Operators always [], eventually $<>$ and until $U$ are some of the modal operators of LTL supported by sPIN. For space reasons, since until is not used in this paper we do not describe it. Operators always and eventually are able to express properties that must be 
true along execution traces of the form $\pi=s_{0} \cdot s_{1} \cdot s_{2} \cdots$. Thus, $\pi$ satisfies [] $\Phi$, written as $\pi \models[] \Phi$ when, for all $i \geq 0$, the subtrace of $\pi$ starting at $s_{i}\left(\pi^{i}=s_{i} \cdot s_{i+1} \cdots\right)$ satisfies $\Phi$, that is, when $\forall i \geq 0 . \pi^{i} \models \Phi$. Operator always is commonly used to express invariant or safety properties. For instance, property "variable $x$ is never negative" could be written as [] $(x \geq 0)$. Operator eventually $<>$ is used to describe properties that must be true at least once in the trace. More formally, $\pi \mid=<>\Phi$ when there exists $i \geq 0$ such as $\pi^{i}=\Phi$. Thus, eventually is weaker than always in the sense that it is easier to be held in a trace. Operator eventually is commonly used to describe the so-called liveness properties, that is, properties that imply some progress in the system. For instance, property "process $P$ executes sentence labelled by $l b$ eventually" can be written as $<>(P @ l b)$. In many occasions, operators always and eventually are combined to describe more complex properties. For example, pattern [] $(p-><>q)$ represents a typical liveness property in LTL. It means that "each occurrence of $p$ in a trace must eventually be followed by an occurrence of $q$ ".

Finally, it is important to remark that all LTL formulas are universally quantified in an implicit manner. This means that when SPIN is given a PROMELA model $M$ and a property (written as an LTL formula $\Phi$ ), it analyzes $\Phi$ on all behaviours of $M$ (all the different traces produced by its execution). If SPIN finds a trace $\pi$ which does not satisfy $\Phi$, it returns $\pi$ as a counterexample of the property.

\subsection{The PRomela Models for MTIP}

We follow an incremental approach to modeling and verification with two models of MTIP that exhibit different levels of abstraction. The first model (A) is suitable to verify properties P1,P2 and P3. The second model (B) is oriented to property $\mathrm{P} 4$, but also can be used for the others. The structure of the two models is represented in Figure 3. Both models contain the two processes sender and seceiver, two unidirectional communication channels representing two sublinks and one application sending user data (process Source). Note that the models contain some elements not used in a real implementation, but they are feasible abstractions that do not modify the 's real logic. In particular, we need to address the problem of modeling time in a language that does not support timers but instead the timeout construction to recover from packet lost. We also need to reduce the range of variables like sequence numbers to keep the model verifiable with the available memory. In the following, we provide the details of the two models, the justification of the abstraction decisions and a simulation trace.

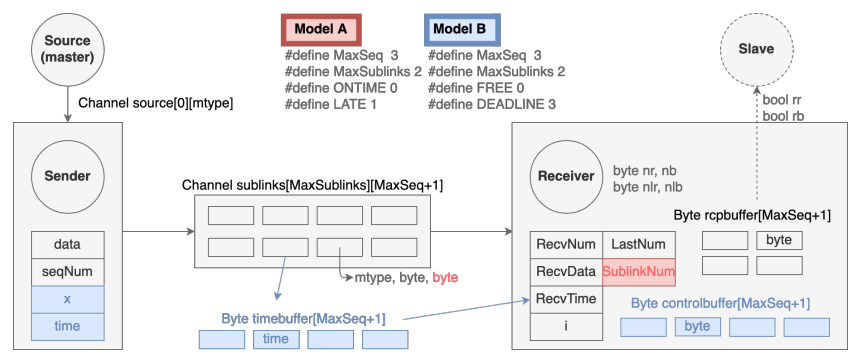

Figure 3: Graphical simplified view of the PROMELA models

\section{Listing 1: Model A (Common variables and sender)}

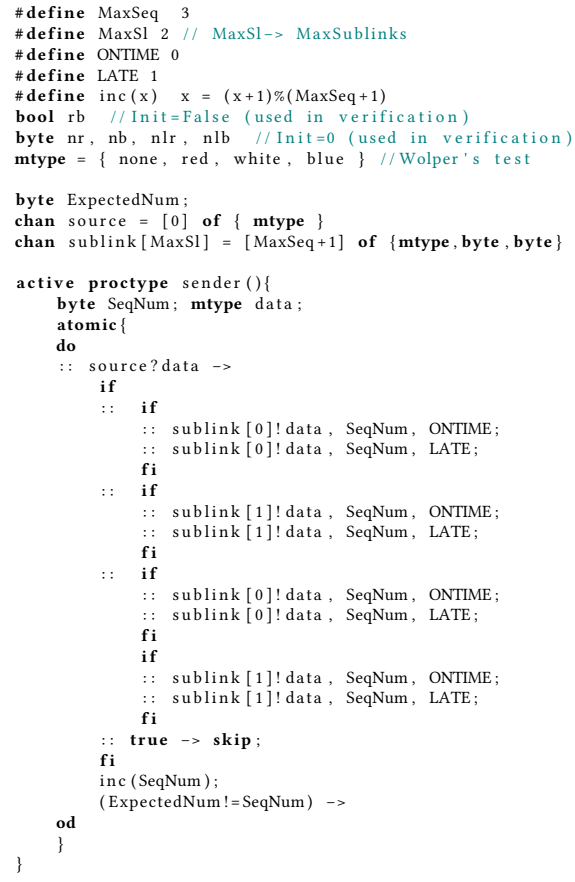

\subsubsection{Description of the model A.}

The main components of the model are the two processes sender and receiver using two unidirectional sublinks. The data structures, constants and the sender process are defined in Listing 1. sender is modeled with a loop getting data from the channel source and sending it in a non-deterministic way using only sublink 0 (lines 20 to 23), only sublink 1 (lines 24 to 27), the two sublinks (lines 28 to 35) or none of them (line 36). Selecting no sublinks when sending is a low cost way to model packet loss. With this nondeterministic selection at the sender, we are offering, in practice, any combination to the receiver. Each packet sent includes its corresponding sequence number (range 0 to 3 ) and the abstraction of the timestamp information (ONTIME or LATE). With the loop in the sender, each packet is managed to have any potential behaviour when travelling in the network, two ONTIME packets being the best case and two lost packets the worst.

\section{Listing 2: Model A (Source)}

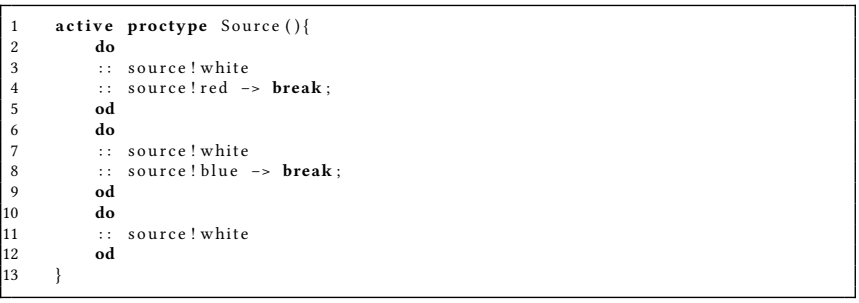

There are some details in the model to save resources during verification, like the use of atomic blocks, the use of the synchronous channel source (Listing 2), or the inclusion of the sentence (ExpectedNum != SeqNum) in line 39 as blocking point. This last 
sentence acts as a guard to suspend the sender process in case of attempt to send a new packet with the same sequence number already in transit to the receiver. This not feasible in a real implementation, but it is a valid abstraction due to the fact that the 64 bits length in the sequence number will never allow the sender to send a new packet with the same sequence number as an active packet in the network (the previous one would already be consumed, discarded at reception due to a late arrival or lost).

\section{Listing 3: Model A (Receiver part 1: Variables and Receiving)}

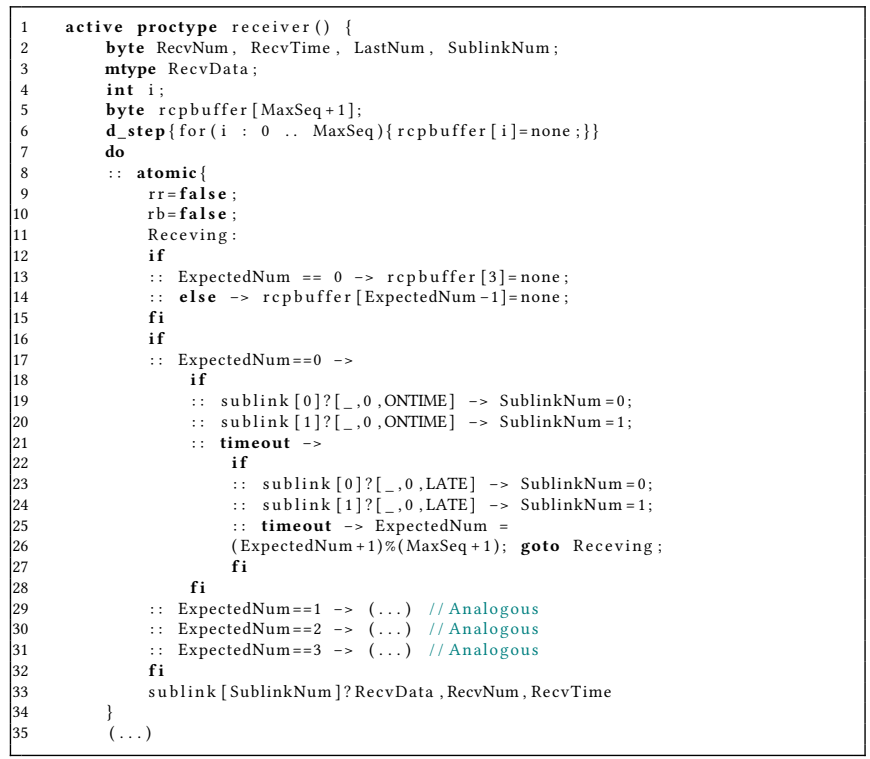

The process receiver executes an infinite loop with two parts. In the first part (Listing 3), it executes one of the 4 blocks depending on the expected sequence number. Each block has the same behaviour as the one in lines 18 to 28: if the expected packet is available ONTIME, the sublink is selected (in a non-deterministic manner if it is available on both). If ONTIME is not available, then the timeout in line 21 moves to read LATE packets. Again, if no LATE packets arrive, a second timeout in line 25 informs on packet lost, increases the counter of the expected sequence number and continues the loop to receive a new packet (line 11).

Listing 4: Model A (Receiver part 2: Algorithm)

if

$\therefore$ if $\operatorname{Rec}$

cvTime $==$ ONTIME $\rightarrow / /$ Case : Ontim

rcpbuffer[RecvNum] != none $\rightarrow / /$ Case: Duplicate

rcpbuffer [RecvNum]=none

rcpbuffer [RecvNum] = RecvData

RecvData $=$ Data

nr++;

assert $(! r b)$; assert $(\mathrm{nl} r==0)$; $\operatorname{assert}(\mathrm{nr}<=1)$

RecvData $==$ blue $->$

$\mathrm{nb}++; \quad \mathrm{rb}=$ true

$::$ assert $($ nlsip $\rightarrow$ skip;
fi

fi

$\begin{gathered}\text { fi } \\ \text { if } \\ \text { if }\end{gathered}->/ /$ Case: Late

if

$\begin{aligned} \text { RecrData } & ==\text { red } \rightarrow \mathrm{nlr}++ \text {; } \\ \text { RecrData } & ==\text { blue } \rightarrow \mathrm{nlb}++\end{aligned}$

f

else $\rightarrow$ skip

fi

1
In the second part (Listing 4), the received packet is processed. The packets on time are inserted in the structure rcpbuffer [] (line 8), in order to control duplicate copies, and they are consumed provided there are no subsequent copies of a previous received packet with the same sequence number (line 9). Note that we also update a number of variables $(\mathrm{nr}, \mathrm{nb}, \mathrm{rb}, \mathrm{nl} r, \mathrm{nlb})$ that refer to the reception of blue or red data and that will only be used for checking properties as described in Section 4.3.

\subsubsection{Description of the model $B$.}

Model B is an alternative to Model A exposing more details on the management of time and potential reorder. Structure timebuffer [] in Model B includes a counter per active sequence number that abstracts the time that a packet has been on the network until it reaches the receiving side. Such counter is increased every time a new packet is sent until a deadline is reached (when no more updates are performed in order to avoid states in the model checking process) or until the packet is consumed by the receiver (when the entries in this structure become free). Listing 5 presents the sender process that always sends the packets using the two sublinks. Listing 6 presents the operation to increase time. The selection of three values as options to increase time (lines 6 to 9) is discussed in Section 4.2.3. The sentence (timebuffer[SeqNum]==FREE) in line 12 of Listing 5 controls that a free slot in the structure is available and could suspend the sender until the receiver frees space.

\section{Listing 5: Model B (New Global Variables and sender)}

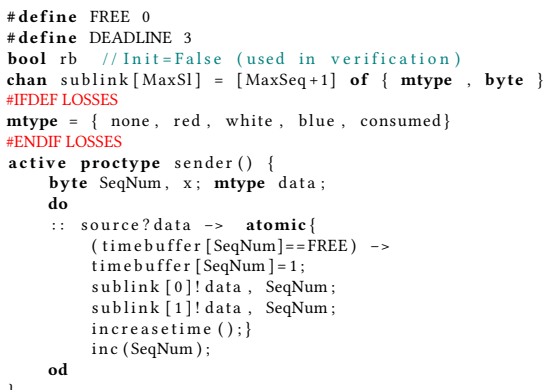

Listing 6: Model B (Modeling time)

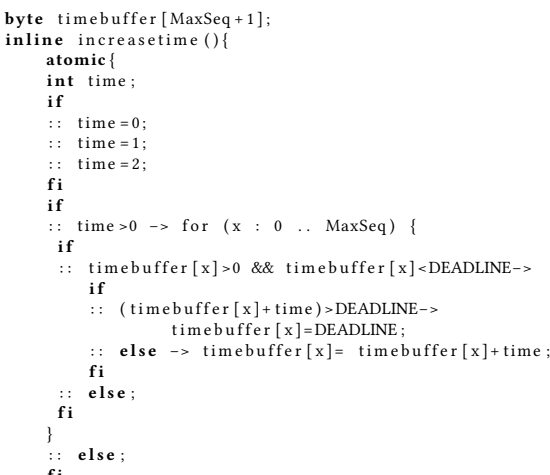

Listing 7 contains the reception on the receiver process. The process executes an infinite loop with a non-deterministic selection of the sublink to read from every iteration (lines 12 to 15). Unlike 
the previous Model A, where the non-deterministic losses were implemented in the sender process, in Model B the possible losses are implemented in the receiver, as shown in Listing 8 . Once the message is read from the channel, the sequence number RecvNum is used to check if the packet is still on time (lines 9 and 41). The packets on time are inserted in the structure rcpbuffer [] (line 22 ) in order to control duplicate copies, and they are consumed provided there are no subsequent copies of a previous received packet with the same sequence number (line 16 and 17).

Listing 7: Model B (Receiver part 1: New Variables and Receiving)

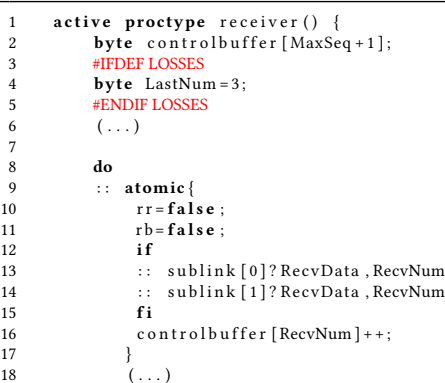

Listing 8: Model B (Receiving part 2: Reception Algorithm)

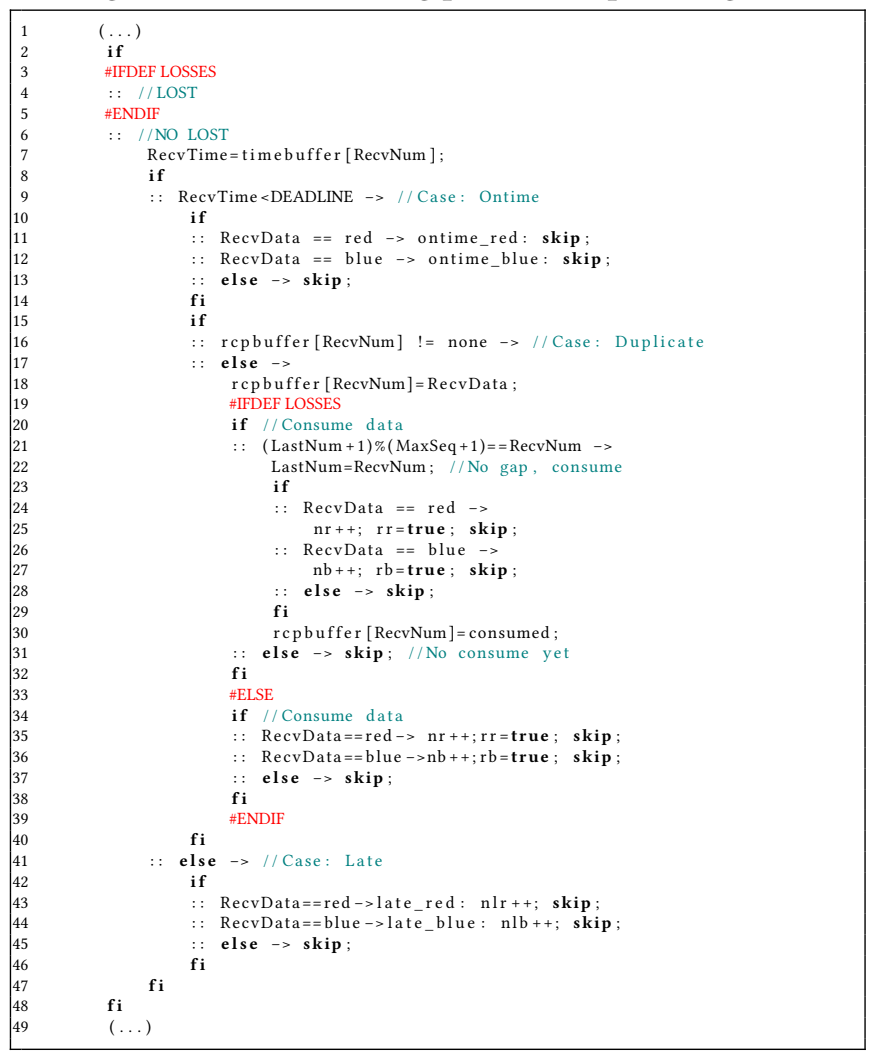

Finally, controlbuffer [] is used to clean the aforementioned rcpbuffer [] and timebuffer[] when any sequence number is going to be reused by the model's sender process (see Listing 9). This structure and control operations such as the one in line 12 of Listing 5 are needed in the model due to the limited amount of numbers available for the sequence numbers. Limitation that will not occur in a real implementation with 64 bits length sequence numbers, as already explained in previous Model A and Section 3.2.

\section{Listing 9: Model B (Receiving part 3: Clean Buffers)}

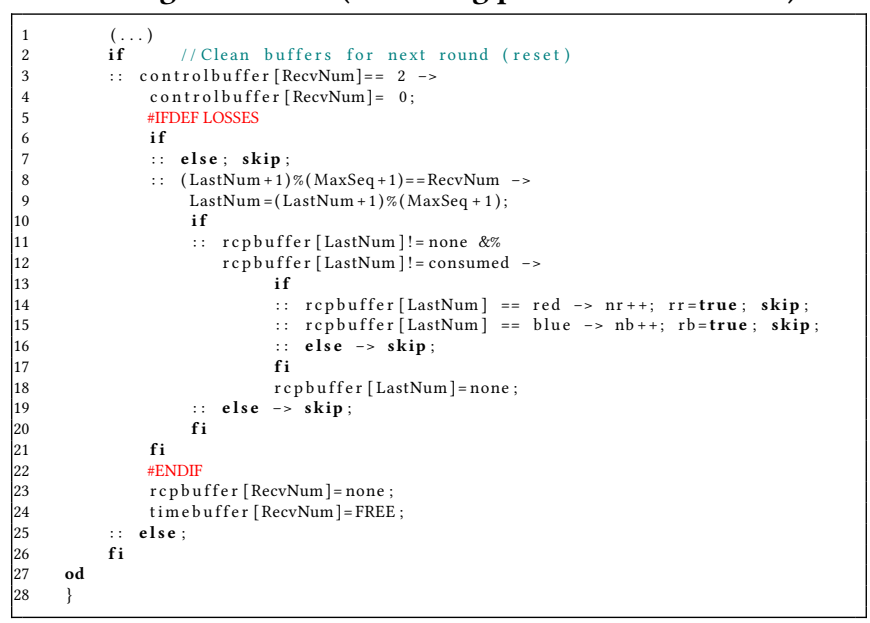

\subsubsection{Abstraction Decisions.}

The main difficulty to use model checking is how to build an abstract model of the protocol that represents the relevant behaviours, but at the same time can be analysed with the available resources (mainly system memory). In our models of MTIP, such abstractions should carefully consider the size of the relevant parameters sequence numbers, number of values for time counters and number of sublinks.

Sequence numbers. A major issue when modeling protocols that use sequence numbers is to fix the actual range of the sequence numbers to ensure that the verification results can be later extrapolated to any range. This problem was already addressed by G. Holzmann in the main paper presenting the model checker sPIN [17], thanks to the reinterpretation of the previous work by P. Wolper [31]. One conclusion in Wolper's work related to the correctness of concurrent systems was the so called "data independence test", that states that verification results are valid for a given set of (user) data if the behavior of the underlying system managing these data does not depend on the specific set. Holzmann translated this result to control flow protocols in this manner: "The question then is, how many different data items do we need to use to obtain a reliable proof? The minimal number turns out to be independent of both the sequence number range and the window size: fust three distinct data items suffice for any flow control protocol, as first shown by Wolper [31]. Traditionally, three different colors are used, e.g., red, white, and blue. The input data sequence consists of one red and one blue message inserted randomly in an infinite sequence of white messages.". We can borrow Wolper's and Holmann's ideas and use their sequences of red, white, and blue data with appropriated sequence numbers, and we fixed sequence numbers in the range 0 to 3 to be reused in a circular manner as usual. Listing 2 includes the code of the Source process defined by Holzmann. This process injects the infinite sequences of user data to the process sender through the channel source. 
Values for time counters. We need to have the same discussion for the values of time stored in the structure buffertime[] in Listing 6 (function increasetime()). The effect of increasing the counter is to locate the packet closer to be discarded by a deadline (also referred as the packet is late). We use three values 1,2 and 3 as the units to represent time ( 0 means that the tag is free and can be reused) and we increase the counter of each active packet in a nondeterministic manner (selecting one of these values) every time a new packet is sent. Value 1 represents no further time progress than the minimum round trip time, modeling ideal speed in the channel for a while. Value 3 means the channel substantially worsens for a while, provoking reaching the deadline (packets arrive late). Value 2 means a chance for normal transmission.

Number of sublinks. The use of several sublinks can provoke packet reorder (unexpected sequence number) and duplicates (same sequence number from several sublinks) at the input of the MTIP receiver process. It is clear that we need at least 2 sublinks to model reorders and duplicates. The question now is whether more than 2 sublinks add significant new reorders of duplicates compared to 2 sublinks, considering the range of sequence numbers. The use of non-deterministic sending in Model A and non-deterministic receiving in Model $\mathrm{B}$, combined with two sublinks is enough to reproduce the needed behaviors in terms of packet lost and reorder due to different speeds in the sublinks. Adding more sublinks would generate longer reorders, however, the reorder generated with two sublinks is enough to check this feature in the protocol.

It is worth noting that all the assumptions above are still valid when the application makes use of awareness to make specific requests to MTIP. For instance, if the application requests only using a subset of the available links, or even just one link, the potential behaviors are a subset of those produced by the PROMELA model with the abstractions described in this section.

\subsubsection{MTIP Simulation With SPIN.}

In Figure 4 we can see how MSCs are generated in SPIN through the promela Models (Model A on the left and Model B on the right). The behavior of the models are the same as previously presented and similarities can be directly comprised with previous MSC provided in Figure 2.

\subsection{Formal Verification}

The verification flow with SPIN starts with the analysis of common concurrency properties like absence of deadlock and other programming errors (use of indexes in arrays, arithmetic operations, etc.). Rows labelled as "No ltl" in Table 2 show the main results in terms of produced states and resources used with different values of the time slots. In all the cases, the two models are correct and no common errors are found. The second step in verification consists in checking specific properties like the ones described as required by MTIP at the beginning of Section 4 . We started with Model A using assertions and then repeated the process with model B using temporal logic. The reasons behind this decision is the cost of checking temporal formulas over Model A due to the huge number of states produced when making the interaction of the internal automata representing the formula and the actual processes. Another

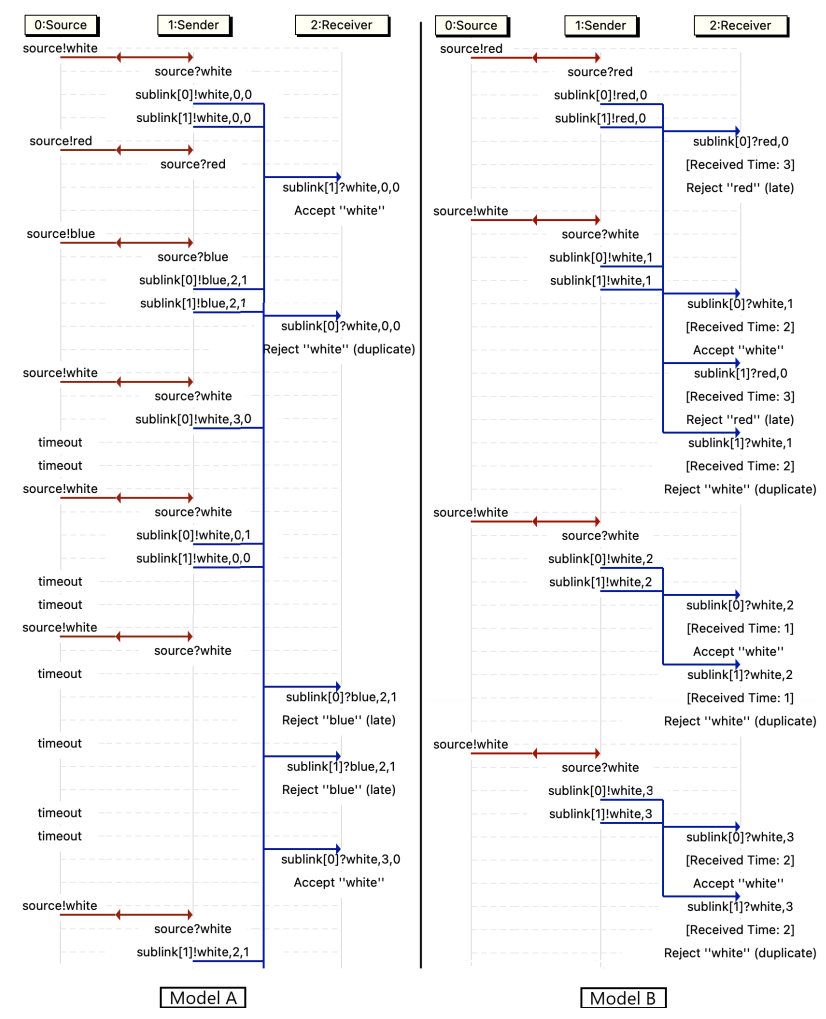

Figure 4: MSCs generated from the PROMELA models by SPIN

practical reason is to first use a more invasive but closer way to define requirements in software designs. In particular, properties P1, P2 and P3 are represented in Model A in lines 12 and 15 of Listing 4. All these properties are satisfied, as reported in Table 2 in the row "Asserts".

Table 2: Verification results

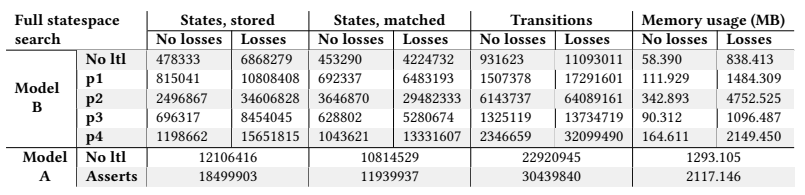

The four properties can be checked over Model B using the temporal formulas in Listing 10. Note that these formulas use the additional variable $r r$ to represent that a red message has been processed by the MTIP protocol and offered to the application and the receiver side, variable equivalent to the $r b$ variable used as well in the previous model for the blue messages. The variables $\mathrm{nb}$, $\mathrm{nlb}$ and $\mathrm{nr}, \mathrm{nlr}$ are used to count such data and the number if late data in each colour. The labels starting with receiver@label are used to check specific sentences in the execution. All these formulas has been successfully verified against the MTIP PROMELA Model B, and the relevant numbers produced by the verification are reported in Table 2 in rows labelled as "p1", "p2", "p3" and "p4", respectively. 
Listing 10: Temporal properties

\begin{tabular}{|c|c|}
\hline 1 & $\left.\begin{array}{lll}1 \mathrm{tl} & \mathrm{p} 1\{[] & {[\mathrm{n}}\end{array}((\mathrm{nr}<=1) \& \&(\mathrm{nb}<=1))\right\}$ \\
\hline $\begin{array}{l}2 \\
3\end{array}$ & $\begin{array}{l}\text { \#FFDEF LOSSES } \\
1 \mathrm{t} 1 \mathrm{p} 2\{[\mathrm{n} l \mathrm{r}==2 \rightarrow[](\mathrm{nr}==0)) \& \&[] \quad(\mathrm{nlb}==2 \rightarrow[](\mathrm{nb}==0))\}\end{array}$ \\
\hline 4 & 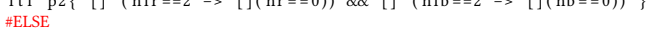 \\
\hline 5 & 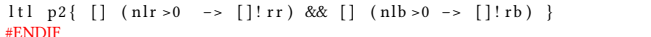 \\
\hline 7 & $1 \mathrm{tl} p 3\{[](\mathrm{rb} \rightarrow[] ! \mathrm{rr})\}$ \\
\hline $\begin{array}{l}8 \\
9\end{array}$ & 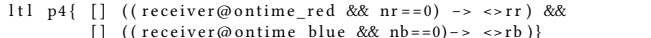 \\
\hline
\end{tabular}

\section{CONCLUSIONS AND FUTURE WORK}

MTIP uses multi-connectivity in multi-homed Tactile Internet devices connected to different wireless networks to increase reliability and reduce latency through the selection of the most reliable and fastest paths. The real-time nature of data in this application domain suggest to avoid flow control mechanisms in favor of low latency. The main issue is how to deal with sequence numbers, timestamps and multiple links to generate an ordered flow of data to the receiver. In the paper, we have presented two formal models of the protocol and the verification work that ensures their correctness. Future work will address performance aspects in different wireless scenarios, including both models with timed automata and our real network and tools described in [10] and [12].

\section{ACKNOWLEDGMENTS}

Work supported by the 5Genesis Project (European Union H2020 research and innovation grant agreement No. 815178) and by the Ministry of Education of Spain (grants FPU17/04292 and RTI2018099777-B-I00). The authors also thank Anna Brunström for the discussions during the internship of Delia Rico at Karlstad University.

\section{REFERENCES}

[1] 3GPP. 2017. 3rd Generation Partnership Project; Technical Specification Group Services and System Aspects; Service requirements for the $5 \mathrm{G}$ system; Stage 1 (Release 16). Technical Report 22.261. 3rd Generation Partnership Project. Version 16.0.0.

[2] 3GPP. 2020. 3rd Generation Partnership Project; Technical Specification Group Services and System Aspects; Service requirements for the $5 \mathrm{G}$ system; Stage 1 (Release 18). Technical Report 22.261. 3rd Generation Partnership Project. Version 18.0.0.

[3] 3GPP. 2021. 3rd Generation Partnership Project; Technical Specification Group Core Network and Terminals; General Packet Radio System (GPRS) Tunnelling Protocol User Plane (GTPv1-U) (Release 17). Technical Report 29.281. 3rd Generation Partnership Project. Version 17.0.0.

[4] 3GPP. 2021. 3rd Generation Partnership Project; Technical Specification Group Services and System Aspects; Service requirements for cyber-physical control applications in vertical domains; Stage 1 (Release 18). Technical Specification (TS) 22.104. 3rd Generation Partnership Project. Version 18.0.0.

[5] M. Amend and D. Hugo. 2020. Multipath sequence maintenance. Internet-Draft draft-amend-iccrg-multipath-reordering-01. IETF Secretariat. http://www.ietf. org/internet-drafts/draft-amend-iccrg-multipath-reordering-01.txt http://www. ietf.org/internet-drafts/draft-amend-iccrg-multipath-reordering-01.txt.

[6] Y. Cai and D. Qi. 2015. Control protocols design for cyber-physical systems. In 2015 IEEE Advanced Information Technology, Electronic and Automation Control Conference (IAEAC). 668-671. https://doi.org/10.1109/IAEAC.2015.7428638

[7] A. Cen, M. W. Mutka, D. Zhu, and N. Xi. 2005. Supermedia Transport for Teleoperations over Overlay Networks. In NETWORKING 2005: Networking Technologies, Services, and Protocols (Lecture Notes in Computer Science, Vol. 3462). Springer, 1409-1412. https://doi.org/10.1007/11422778_126

[8] S. Chouali, A. Boukerche, A. Mostefaoui, and M. A. Merzoug. 2020. Formal Verification and Performance Analysis of a New Data Exchange Protocol for Connected Vehicles. IEEE Transactions on Vehicular Technology 69, 12 (2020), 15385-15397. https://doi.org/10.1109/TVT.2020.3040817

[9] Cisco. 2014. Intent-Based Networking (IBN). https://www.cisco.com/c/en/us solutions/intent-based-networking.html. Online; accessed 28 May 2021.

[10] A. Díaz-Zayas, G. Caso, Ö. Alay, P. Merino, A. Brunström, D. Tsolkas, and H. Koumaras. 2020. A Modular Experimentation Methodology for 5G Deployments The 5GENESIS Approach. Sensors 20, 22 (2020), 6652. https://doi.org/10.3390/ s20226652
[11] C. Dombrowski, S. Junges, J. P. Katoen, and J. Gross. 2016. Model-Checking Assisted Protocol Design for Ultra-reliable Low-Latency Wireless Networks. In 2016 IEEE 35th Symposium on Reliable Distributed Systems (SRDS). 307-316. https://doi.org/10.1109/SRDS.2016.048

[12] A. R. Espada, M. Gallardo, A. Salmerón, L. Panizo, and P. Merino. 2019. A formal approach to automatically analyse extra-functional properties in mobile applications. Softw. Test. Verification Reliab. 29, 4-5 (2019). https://doi.org/10. 1002/stvr.1699

[13] O. Holland et al. 2019. The IEEE 1918.1 "Tactile Internet" Standards Working Group and its Standards. Proc. IEEE 107, 2 (Feb 2019), 256-279.

[14] A. Ford, C. Raiciu, M. Handley, and O. Bonaventure. 2020. TCP Extensions for Multipath Operation with Multiple Addresses. RFC 8684. RFC Editor. http: //www.rfc-editor.org/rfc/rfc8684.txt.

[15] V. Gokhale, O. Dabeer, and S. Chaudhuri. 2013. HoIP: Haptics over Internet Protocol. In 2013 IEEE International Symposium on Haptic Audio Visual Environments and Games (HAVE). 45-50. https://doi.org/10.1109/HAVE.2013.6679609

[16] O. Holland, E. Steinbach, R. V. Prasad, Q. Liu, Z. Dawy, A. Aijaz, N. Pappas, K. Chandra, V. S. Rao, S. Oteafy, M. Eid, M. Luden, A. Bhardwaj, X. Liu, J. Sachs, and J. Araújo. 2019. The IEEE 1918.1 "Tactile Internet" Standards Working Group and its Standards. Proc. IEEE 107, 2 (2019), 256-279. https://doi.org/10.1109/JPROC. 2018.2885541

[17] G. J. Holzmann. 1997. The Model Checker SPIN. IEEE Trans. Softw. Eng. 23, 5 (May 1997), 279-295. https://doi.org/10.1109/32.588521

[18] J. Iyengar and I. Swett. 2015. QUIC: A UDP-Based Secure and Reliable Transport for HTTP/2. Internet-Draft draft-tsvwg-quic-protocol-00. IETF Secretariat. https: //datatracker.ietf.org/doc/html/draft-tsvwg-quic-protocol-00

[19] K. S. Kim, D. K. Kim, C. Chae, S. Choi, Y. Ko, J. Kim, Y. Lim, M. Yang, S. Kim, B. Lim, K. Lee, and K. L. Ryu. 2019. Ultrareliable and Low-Latency Communication Techniques for Tactile Internet Services. Proc. IEEE 107, 2 (2019), 376-393. https: //doi.org/10.1109/JPROC.2018.2868995

[20] G. Kokkonis, K. Psannis, M. Roumeliotis, and S. Kontogiannis. 2012. A Survey of Transport Protocols for Haptic Applications. Proceedings of the 16th Panhellenic Conference on Informatics, 192-197. https://doi.org/10.1109/PCi.2012.54

[21] D. Mills, J. Martin, J. Burbank, and W. Kasch. 2010. Network Time Protocol Version 4: Protocol and Algorithms Specification. RFC 5905. RFC Editor. http://www.rfceditor.org/rfc/rfc5905.txt

[22] J. Qadir, A. Ali, K. A. Yau, A. Sathiaseelan, and J. Crowcroft. 2015. Exploiting the Power of Multiplicity: A Holistic Survey of Network-Layer Multipath. IEEE Communications Surveys Tutorials 17, 4 (Fourthquarter 2015), 2176-2213. https: //doi.org/10.1109/COMST.2015.2453941

[23] D. Rico and P. Merino. 2020. A Survey of End-to-End Solutions for Reliable LowLatency Communications in 5G Networks. IEEE Access 8 (2020), 192808-192834. https://doi.org/10.1109/ACCESS.2020.3032726

[24] H. Schulzrinne, S. Casner, R. Frederick, and V. Jacobson. 2003. RTP: A Transport Protocol for Real-Time Applications. STD 64. RFC Editor. http://www.rfc-editor. $\mathrm{org} / \mathrm{rfc} / \mathrm{rfc} 3550$. txt http://www.rfc-editor.org/rfc/rfc3550.txt.

[25] V. Shankarkumar, L. Montini, T. Frost, and G. Dowd. 2017. Precision Time Protocol Version 2 (PTPv2) Management Information Base. RFC 8173. RFC Editor.

[26] V. Singh, T. Karkkainen, J. Ott, S. Ahsan, and L. Eggert. 2014. Multipath RTP (MPRTP). Internet-Draft draft-singh-avtcore-mprtp-10. IETF Secretariat. http: //www.ietf.org/internet-drafts/draft-singh-avtcore-mprtp-10.txt http://www. ietf.org/internet-drafts/draft-singh-avtcore-mprtp-10.txt.

[27] T. Viernickel, A. Froemmgen, A. Rizk, B. Koldehofe, and R. Steinmetz. 2018. Multipath QUIC: A Deployable Multipath Transport Protocol. In 2018 IEEE International Conference on Communications. 1-7. https://doi.org/10.1109/ICC.2018.8422951

[28] C. West. 1978. General technique for communications protocol validation. Ibm fournal of Research and Development 22 (1978), 393-404.

[29] A. Williams, K. Gross, R. van Brandenburg, and H. Stokking. 2014. RTP Clock Source Signalling. RFC 7273. RFC Editor.

[30] R. Wirz, M. Ferre, R. Marín, J. Barrio, J. M. Claver, and J. Ortego. 2008. Efficient Transport Protocol for Networked Haptics Applications. In Haptics: Perception, Devices and Scenarios, M. Ferre (Ed.). Springer Berlin Heidelberg, Berlin, Heidelberg, 3-12.

[31] P. Wolper. 1986. Expressing Interesting Properties of Programs in Propositional Temporal Logic. Association for Computing Machinery, New York, NY, USA, 184-193. https://doi.org/10.1145/512644.512661

[32] T. Zhang, S. Zhao, and B. Cheng. 2020. Multipath Routing and MPTCP-Based Data Delivery Over Manets. IEEE Access 8 (2020), 32652-32673. https://doi.org/ 10.1109/ACCESS.2020.2974191

[33] M. F. Zhani and H. ElBakoury. 2020. FlexNGIA: A Flexible Internet Architecture for the Next-Generation Tactile Internet. Fournal of Network and Systems Management 28, 4 (Mar 2020), 751-795. https://doi.org/10.1007/s10922-020-09525-0 